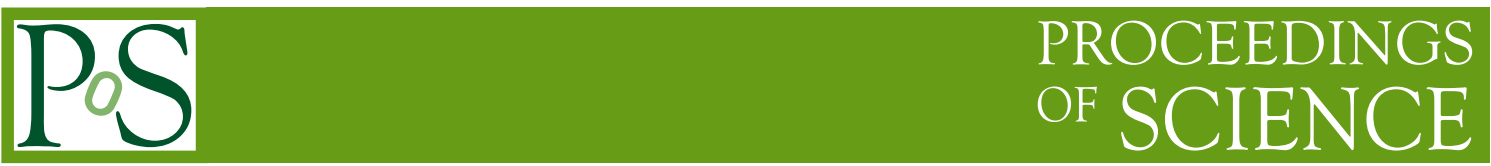

\title{
Fully Hadronic Diboson Searches in ATLAS
}

\author{
Steven Schramm ${ }^{a, *}$, on behalf of the ATLAS Collaboration \\ ${ }^{a}$ Université de Genève, \\ 24 quai Ernest-Ansermet, Geneva, Switzerland \\ E-mail: steven.schramm@cern.ch
}

Fully hadronic diboson searches are a promising means of searching for new physics due to the large number of theoretical models predicting the existence of new mediators which couple to pairs of bosons, and because of the large hadronic branching fractions of Standard Model W, Z, and Higgs bosons. ATLAS has conducted three fully-hadronic diboson resonance searches using the full Run 2 dataset of $139 \mathrm{fb}^{-1}$ of proton-proton collisions delivered by the LHC, aimed at divector-boson (WW/WZ/ZZ), vector boson plus Higgs (WH/ZH), and generic di-boson-like final states. The three searches have complementary sensitivity, and in particular the generic di-bosonlike final state has weaker sensitivity for any specific model as it is instead designed to consider a wide range of possible new physics scenarios that are beyond the domain of acceptance of the other two dedicated searches. No significant deviations beyond the Standard Model expectation were observed in any of the searches, and thus limits are set on a variety of new physics models.

40th International Conference on High Energy physics - ICHEP2020

July 28 - August 6, 2020

Prague, Czech Republic (virtual meeting)

\footnotetext{
${ }^{*}$ Speaker
} 


\section{Introduction}

Searches for new physics are a key part of the ATLAS [1] physics program. There are many theories of new physics beyond the Standard Model (SM) which predict decays of new particles to pairs of SM bosons, motivating searches for both vector-boson pairs (WW, WZ, and ZZ; collectively VV) and vector bosons together with a Higgs boson (WH and ZH, collectively VH). Such SM boson pairs very often result in the production of fully-hadronic final states, thanks to large hadronic branching fractions: $67.41 \%$ for $\mathrm{W}$ [2], 69.911\% for $\mathrm{Z}$ [2], and $58 \%$ for $\mathrm{H} \rightarrow \mathrm{bb}$ [3]. Fully-hadronic diboson analyses are thus a promising means of searching for the rare production of new particles, particularly at the highest energy regimes accessible at the Large Hadron Collider (LHC) where statistical limitations dominate the sensitivity to new physics.

ATLAS has completed three searches for fully-hadronic diboson resonances using the full LHC Run $2 p p$ collision dataset, corresponding to an integrated luminosity of $139 \mathrm{fb}^{-1}$. These analyses have been optimized for three classes of di-boson searches: di-vector-boson resonances (VV) [4], vector boson plus Higgs resonances (VH) [5], and generic di-boson-like resonances [6].

\section{Di-vector-boson resonances (VV)}

Di-vector-boson resonances are sensitive to new physics resulting in the production of massive spin-0 (WW/ZZ), spin-1 (WW/WZ), and spin-2 (WW/ZZ) mediators. In order to have sensitivity to such resonances, the analysis must suppress the enormous QCD multijet production cross-section, which is typically done through the use of jet taggers. Jet taggers use large- $R$ jets with $R=1.0$ to capture the full decays of the V-bosons, and then use the energy and angular structure of the constituents from which the jet is built (the jet substructure) to identify those consistent with the hadronic decay of a V-boson while rejecting those which appear to originate from individual light-quarks or gluons. Requiring two V-tagged jets in an event, combined with analysis selections requiring that the pair of jets are roughly balanced and consistent with s-channel production, accepts 5-10\% of signal events while only accepting 1 in $10^{5}$ background events as shown in Figure 1.
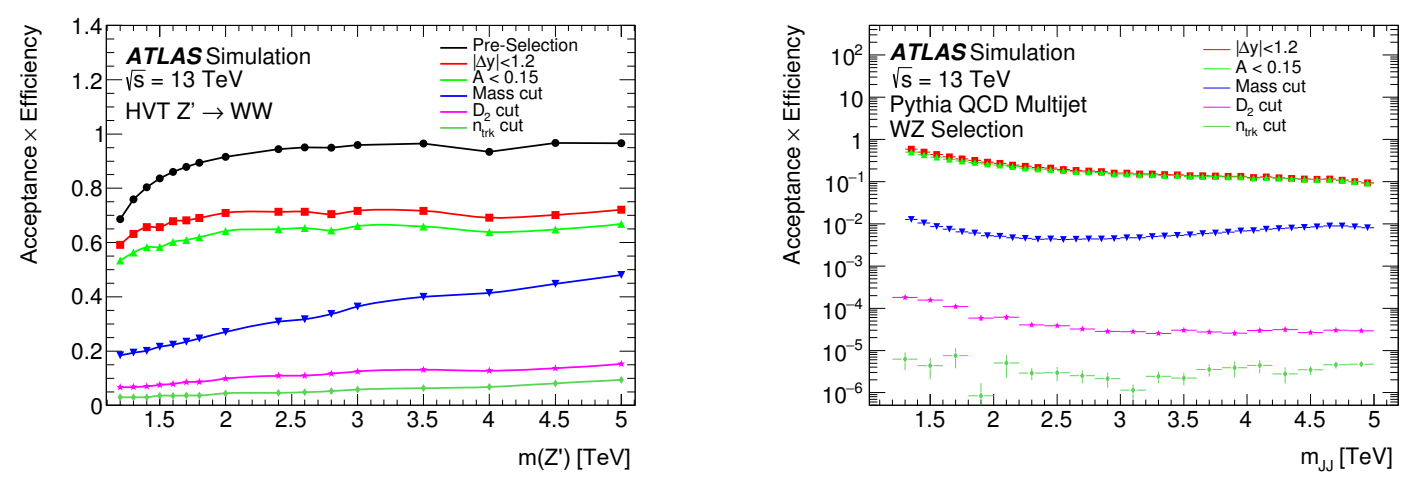

Figure 1: The di-vector-boson analysis selection for signal (left) and background (right) events, demonstrating the enormous background rejection that comes with applying the V-boson tagger (mass, $D_{2}$, and $\mathrm{n}_{\text {trk }}$ cuts) [4].

In order to ensure that the taggers are using data features that are well-modelled in simulated events, a V+jets control region is used to extract the tagging efficiency as shown in Figure 2, thus 
deriving tagger scale factors. After the full analysis selection, the smoothly falling background is fit using a functional form. Figure 2 shows the resulting data spectrum; no significant deviations are observed over the background fit, thus limits are set as will be shown in Section 5.
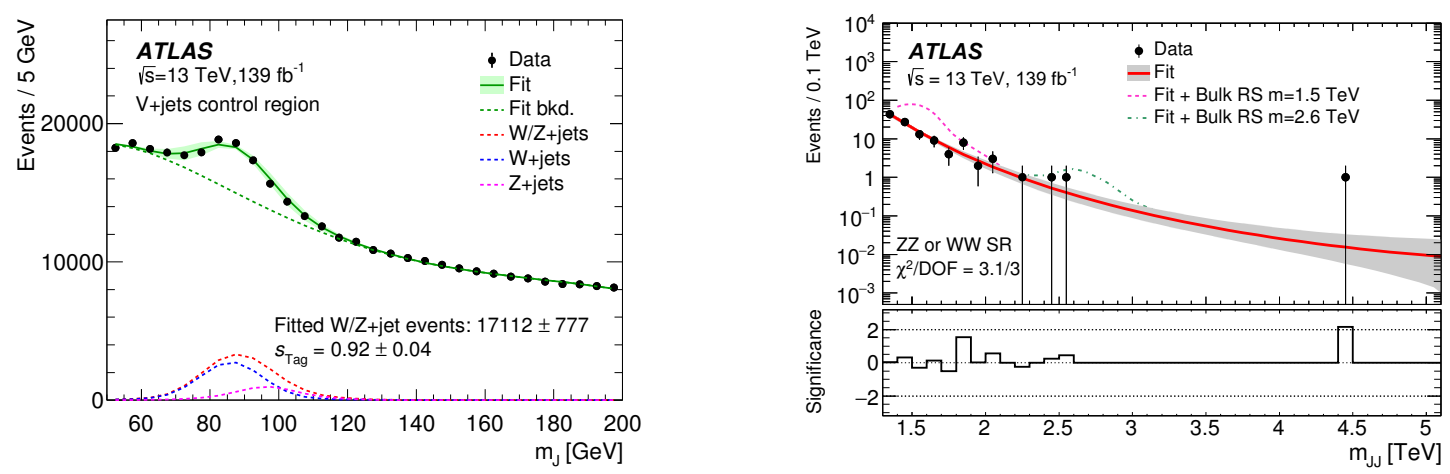

Figure 2: The data/MC agreement of the V-boson tagger used in the di-vector-boson analysis is evaluated in a dedicated $\mathrm{V}+$ jet control region (left). The subsequent application of the full selection to the combined WW or ZZ signal region shows no significant localized excess beyond the smoothly falling background (right) [4].

\section{Vector boson plus Higgs resonances (VH)}

Vector boson plus Higgs resonances are sensitive to the production of new spin-1 mediators, and face similar experimental challenges to VV resonances. In order to suppress the otherwise overwhelming QCD multijet background, large- $R$ jets are used both for $\mathrm{V}$ - and $\mathrm{H}$-tagging. The V-boson taggers are identical to what was used in the VV analysis, while the H-boson tagger primarily relies on the ability to identify the production of $b$-hadrons. Traditionally, large- $R$ jet taggers have used nearby $R=0.2$ track-jets for $b$-hadron identification; the VH analysis instead made use of refined $b$-hadron taggers and Variable-Radius (VR) track-jets. These VR track-jets dramatically increase the acceptance of collimated di- $b$ production, such as high- $p_{\mathrm{T}} \mathrm{H}$-bosons, as shown in Figure 3. A H $\rightarrow b b$ tagger is then optimized for the analysis, accepting 4-10\% of signal events as shown in Figure 3, depending on whether a single- or double- $b$-tag is required.

The analysis makes use of a series of control and validation regions, based on the masses of the V-boson and $\mathrm{H}$-boson candidate jets, to extrapolate to the signal region. This is done separately for both single- and double- $b$-tag selections, in which the control region dijet mass distributions are reweighted to provide a background expectation in the corresponding signal region. No significant deviations are observed, as shown in Figure 4, and thus limits are set as will be shown in Section 5. This analysis improves beyond only statistical gains over the past $36 \mathrm{fb}^{-1}$ analysis due to the use of improved V-taggers, H-taggers, and the use of VR track-jets for $b$-tagging as shown in Figure 4.

\section{Generic di-boson-like resonances}

The previous two analysis both assume that the new physics particle decays to a pair of SM bosons, either via VV or VH final states. However, the new particle may instead decay to a pair of 

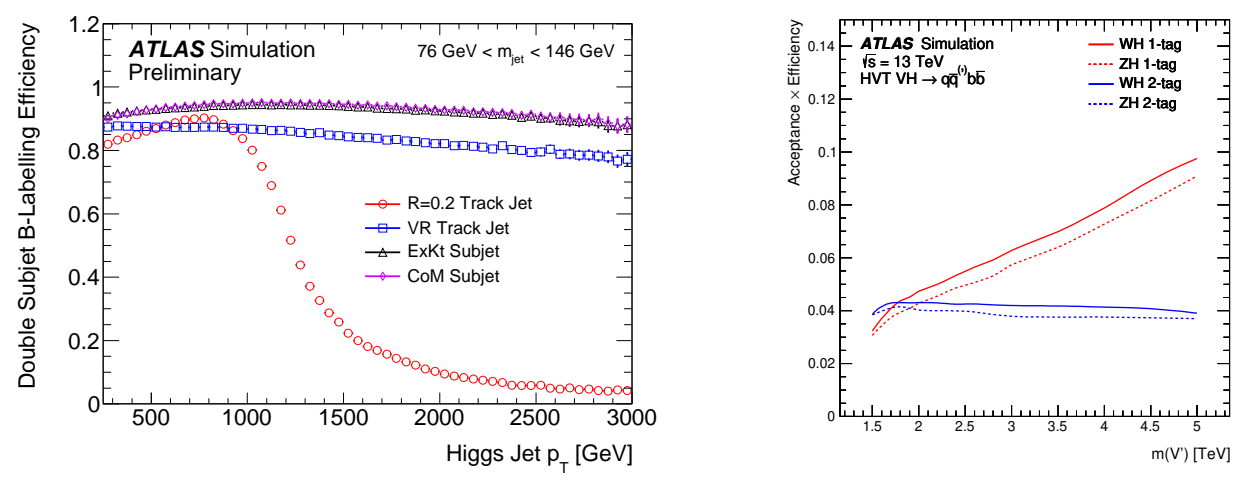

Figure 3: The utility of fixed-radius $R=0.2$ track-jets in $\mathrm{H} \rightarrow b b$-tagging drops dramatically at high $p_{\mathrm{T}}$, which can be recovered using other techniques such as Variable-Radius (VR) track-jets (left) [7]. VR track-jets are then used in the optimization of $\mathrm{H} \rightarrow b b$ taggers for the vector boson plus Higgs analysis (right) [5].
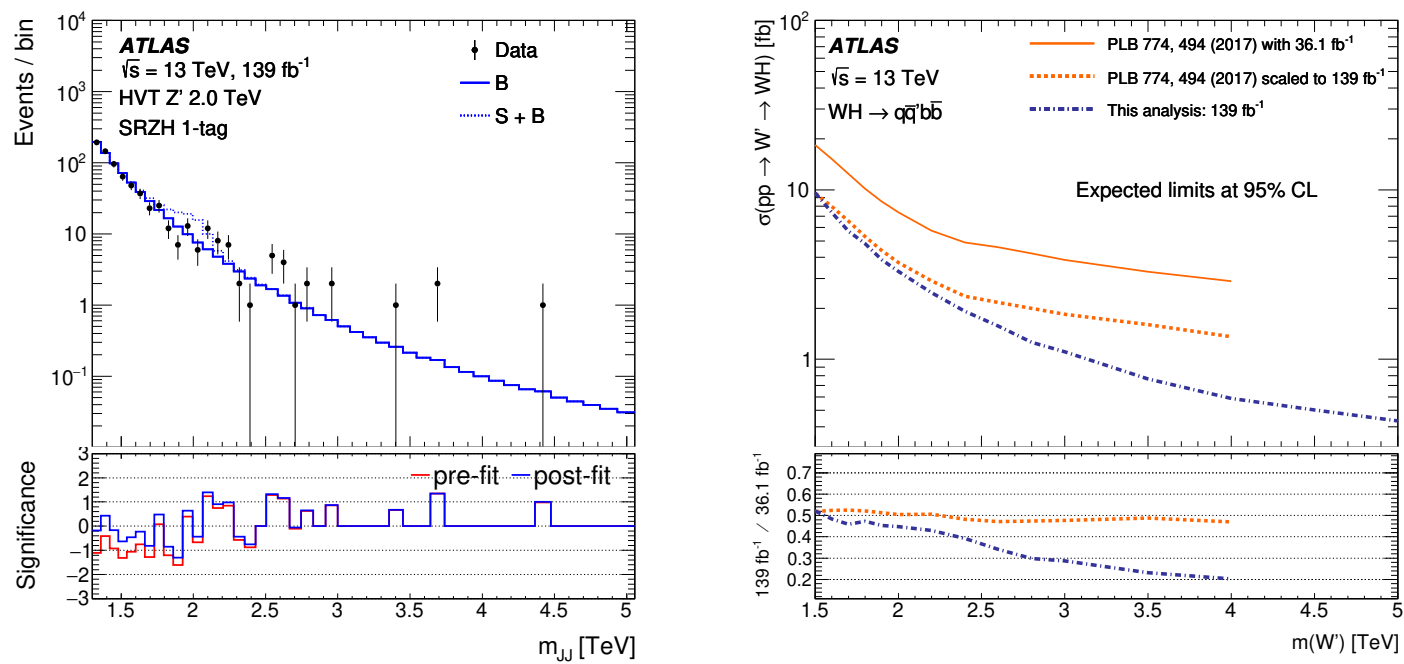

Figure 4: The application of the 1-b-tag optimized selection in the vector boson plus Higgs analysis shows no localized excess beyond the background expectation (left). The analysis sensitivity has been improved substantially through improved V-taggers, H-taggers, and VR $b$-tagging (right) [5].

other new particles $\mathrm{X}$ and $\mathrm{Y}$, which then decay back to pairs of SM quarks resulting in di-boson-like signatures. The di-boson-like resonance search aims at such exotic final states, where the $\mathrm{X}$ and Y particles may independently be either SM or non-SM bosons. This generic search is enabled through the use of neural networks, which are trained directly on data thus avoiding the need to assume a given SM signature. This training proceeds by dividing the large- $R$ dijet mass spectrum into several regions, where at any time a given region is considered to be a signal region and the two adjacent regions are defined to be sidebands. The networks are then trained to differentiate between the current signal region and its sidebands, based only on the masses of the two large- $R$ jets in the event, thus training the network to learn a proxy for the classification of signal. Cuts can then be applied to these networks, enhancing the signal-purity of the region under study if any signal is present. A schematic demonstrating this strategy is presented in Figure 5. 


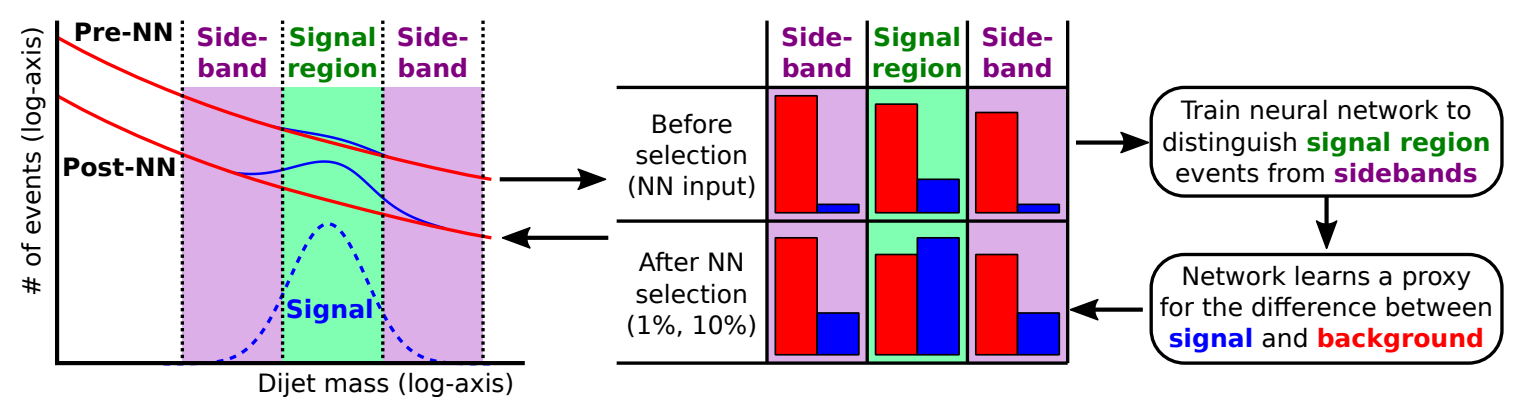

Figure 5: A schematic outlining the analysis methodology of the generic di-boson-like analysis, showing how sidebands are used to train a neural network classifier directly on data thus enabling a generic search.

The application of this procedure using the networks to accept the $10 \%$ of events in the signal region that are more signal-like than sideband-like is shown in Figure 6. The resulting spectrum is generically sensitive to di-boson-like resonances, and is expected to have steps at region boundaries as spectrum is stitched together from separate regions in which the neural network training is performed independently. In case a given signal interpretation is of particular interest, it is possible to re-train the neural networks on a mixture of data and injected simulated signal, thus biasing the network towards the specific injected model. This is also shown in Figure 6, under a signal model interpretation that allows comparison with the two dedicated analyses. No significant deviations are observed in any of the regions studied, and thus limits are set as will be shown in Section 5 .
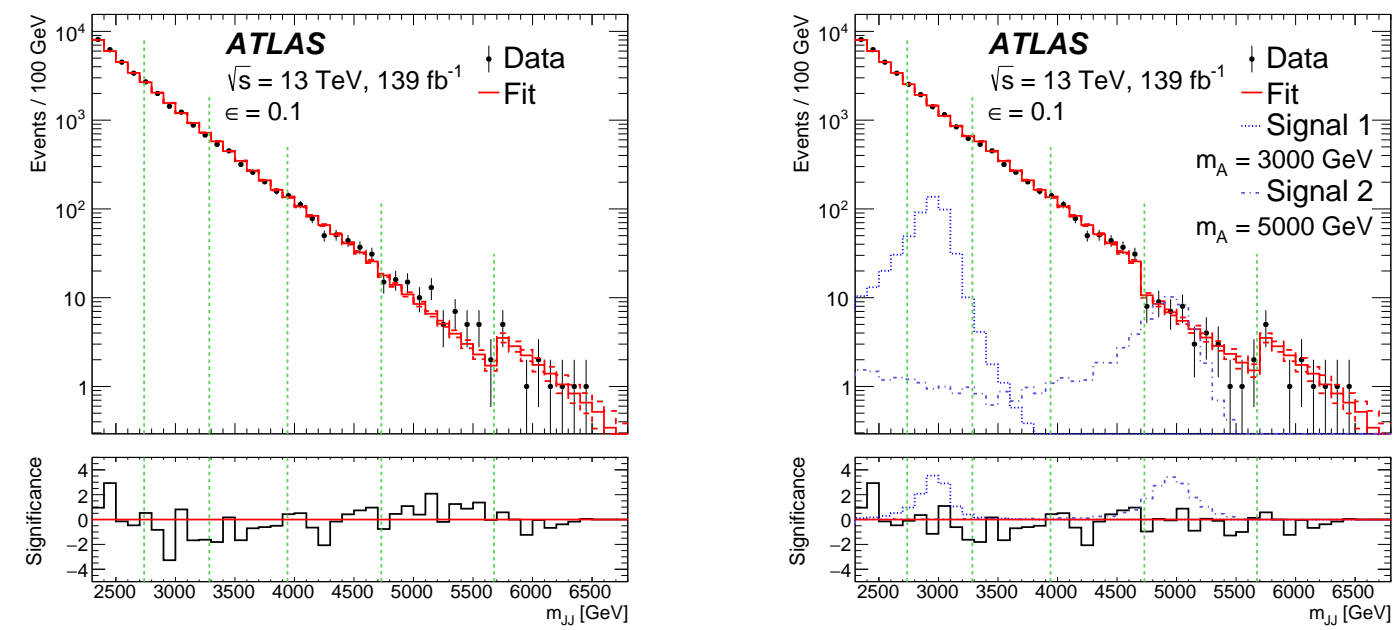

Figure 6: Neural network classifiers are trained both purely on data (left) and on a mixture of data and injected signal models (right), resulting in an analysis that is sensitive to generic new di-boson-like resonances or to specific new physics models, respectively. No significant excesses are observed in either training scenario [6].

\section{Limits on new physics}

The di-vector-boson, vector boson plus Higgs, and generic di-boson-like resonance searches are complementary in the search for new physics. They are sensitive to different possible final states, and can be used to study a diverse set of models of new physics. In final states where they can 
be directly compared, the two dedicated searches perform similarly while the generic search is less sensitive, as shown in Figure 7. This is unsurprising as the generic search is not designed to provide ultimate sensitivity for a specific model, rather it surveys a much wider range of possibilities.
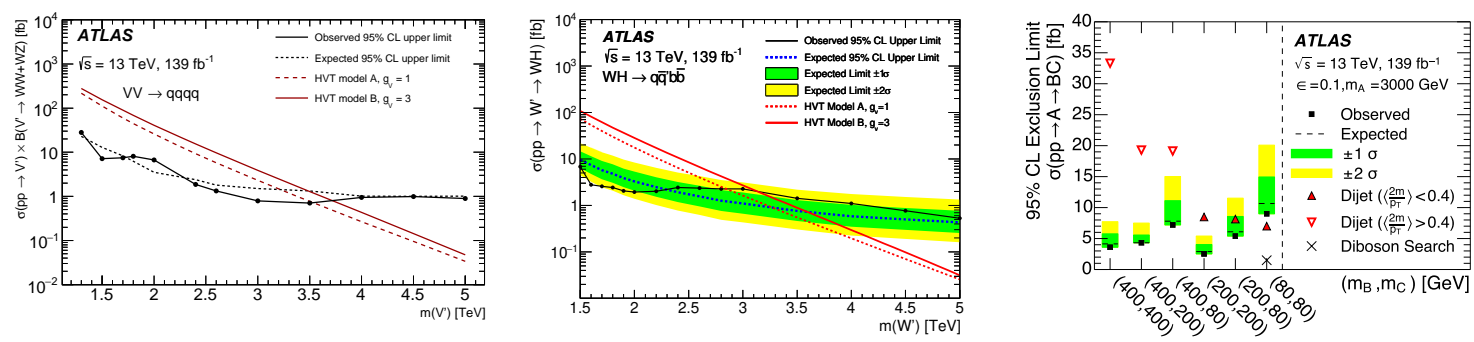

Figure 7: The di-vector boson (left) [4], vector boson plugs Higgs (middle) [5], and generic di-boson-like (right) [6] analyses provide complementary limits in the search for new physics.

\section{Summary}

ATLAS has conducted three fully-hadronic searches for new physics resulting in di-boson final states using the full LHC Run 2 dataset of $139 \mathrm{fb}^{-1}$ of $p p$ collisions. These three searches cover final states including the production of WW, WZ, ZZ, WH, and ZH boson pairs, as well as a generic search for di-boson-like production where the new particle does not have to decay to Standard Model bosons. No significant deviations from the Standard Model background expectation have been observed, and thus the analyses set complementary limits on a range of models of new physics.

\section{References}

[1] ATLAS collaboration, ATLAS Experiment at the CERN Large Hadron Collider, JINST 3 (2008) 003.

[2] P. D. Group, P. A. Zyla, R. M. Barnett, J. Beringer, O. Dahl, D. A. Dwyer et al., Review of Particle Physics, Progress of Theoretical and Experimental Physics 2020 (08, 2020) .

[3] LHC Higgs Cross Section Working Group, Handbook of LHC Higgs Cross Sections: 4. Deciphering the Nature of the Higgs Sector, 1610.07922.

[4] ATLAS collaboration, Search for diboson resonances in hadronic final states in $139 \mathrm{fb}^{-1}$ of pp collisions at $\sqrt{s}=13 \mathrm{TeV}$ with the ATLAS detector, JHEP 09 (2019) 091, [1906.08589].

[5] ATLAS collaboration, Search for resonances decaying into a weak vector boson and a Higgs boson in the fully hadronic final state produced in proton-proton collisions at $\sqrt{s}=13 \mathrm{TeV}$ with the ATLAS detector, 2007.05293.

[6] ATLAS collaboration, Dijet resonance search with weak supervision using $\sqrt{s}=13 \mathrm{TeV} p p$ collisions in the ATLAS detector, Phys. Rev. Lett. 125 (2020) 131801, [2005. 02983].

[7] ATLAS collaboration, Variable Radius, Exclusive- $k_{T}$, and Center-of-Mass Subjet Reconstruction for Higgs $(\rightarrow$ b $\bar{b})$ Tagging in ATLAS, ATL-PHYS-PUB-2017-010 (2017) . 